\title{
Criação de tilápias no efluente doméstico tratado em lagoas de estabilização
}

\author{
Clovis Matheus Pereira ${ }^{1}$ \\ Flavio Rubens Lapolli² \\ Universidade Federal do Recôncavo da Bahia, C.C.A.A.B, Campus Universitário \\ CEP 44 380-000, Cruz das Almas - BA, Brasil \\ ${ }^{2}$ Departamento de Engenharia Sanitária e Ambiental, Centro Tecnológico \\ Universidade Federal de Santa Catarina, Florianópolis - SC, Brasil \\ *Autor para correspondência \\ clovismatheuspereira@hotmail.com
}

Submetido em 26/02/2008

Aceito para publicação em 05/10/2008

\section{Resumo}

A capacidade de produção e a qualidade da carne das tilápias (Oreochromis niloticus) criadas no efluente de lagoas de estabilização foram testadas nas densidades de 3 peixes $/ \mathrm{m}^{2}\left(\mathrm{~T}_{2}\right)$ e 7 peixes $/ \mathrm{m}^{2}\left(\mathrm{~T}_{3}\right)$ e a criação de 7 peixes $/ \mathrm{m}^{2}$ em água limpa + ração( $\left.\mathrm{T}_{1}\right)$ utilizando 3 repetições para cada tratamento em tanques de $2,57 \mathrm{~m}^{2} \mathrm{e}$ $0,60 \mathrm{~m}$ de coluna d'água com aeração tipo airlift. Os peixes criados em água limpa e ração $\left(\mathrm{T}_{1}\right)$ cresceram mais. A maior densidade do $\mathrm{T}_{3}\left(7 / \mathrm{m}^{2}\right)$ com relação ao $\mathrm{T}_{2}\left(3 / \mathrm{m}^{2}\right)$ não proporcionou diferença na produção total final ( $p>0,05)$, compensado pelo maior crescimento individual dos peixes em menor densidade. As condições para sobrevivência dos peixes no efluente da ETE são atestadas pela alta sobrevivência $(>90 \%)$ nos tratamentos $\mathrm{T}_{2}$ e $\mathrm{T}_{3}$; mas as concentrações de $\mathrm{N}$ - amônia total em apenas 10\% das amostras coletadas se mostraram favoráveis ao crescimento dos peixes $(<2,0 \mathrm{mg} / \mathrm{L})$. O sistema de criação de peixes melhorou a qualidade do efluente gerado, reduzindo as concentrações de Nitrogênio orgânico total e diminuindo os sólidos suspensos. As análises de coliformes fecais, Salmonella sp. e Staphylococcus aureus dos efluentes e da carne dos peixes produzidos ficaram dentro dos padrões exigidos pela OMS (Organização Mundial de Saúde).

Unitermos: piscicultura, reuso de água, qualidade de água, tratamento de esgotos

\section{Abstract}

Nile tilapia culture on domestic effluent treated in stabilization ponds. The performance and filet quality of tilapias (Oreochromis niloticus) culture in effluent stabilization lagoons was tested at the densities of $3 \mathrm{fish} / \mathrm{m}^{2}\left(\mathrm{~T}_{3}\right), 7 \mathrm{fish} / \mathrm{m}^{2}\left(\mathrm{~T}_{2}\right)$ and $7 \mathrm{fish} / \mathrm{m}^{2}$ in clean water $+\operatorname{diet}\left(\mathrm{T}_{1}\right)$ with 3 repetitions in tanks of $2.57 \mathrm{~m}^{2}$ and $0.60 \mathrm{~m}$ of water column with supplemental aeration. Fish culture in clean water plus diet $\left(\mathrm{T}_{1}\right)$ presented the highest growth. The higher density $\mathrm{T}_{3}\left(7 / \mathrm{m}^{2}\right)$ compared to $\mathrm{T}_{2}\left(3 / \mathrm{m}^{2}\right)$ did not result in any difference of total production $(\mathrm{p}>0.05)$ but this was compensated by the increased individual fish growth rate at lower density. The conditions that sustain fish survival culture with ETE effluent were attested by the high survival $(>90 \%)$ under both treatments, but only $10 \%$ of the water samples from $\mathrm{T}_{2}$ and $\mathrm{T}_{3} \mathrm{~N}$-total ammonium was favorable for fish growth $(<2.0 \mathrm{mg} / \mathrm{L})$. The rearing system improved the effluent quality, reducing the total organic nitrogen and the solids in suspension. The faecal coliforms, Salmonella sp. and Staphylococcus aureus from the effluent and fish were verified to be within the standards laid down by the World Health Organization (WHO).

Key words: pisciculture, waste reuse, water quality, waste treatment 


\section{Introdução}

Produzir peixes no efluente de uma estação de tratamento de esgoto pode parecer desnecessário em um país, como o Brasil, que tem em grande parte de seu território abundância de água. Os esgotos tratados nos moldes atuais causam impactos ambientais quando utilizam o ambiente natural como depósito do excesso de eutrofização e nutrientes resultantes do tratamento dado ao esgoto, os mesmos nutrientes que são utilizados para produzir alimentos criando uma situação de insustentabilidade, desperdício.

A concepção atual dos sistemas produtivos considera necessária a utilização de Tecnologias Limpas que praticam o combate ao desperdício com estratégias integradas, aplicadas preventivamente aos processos, produtos e serviços, com o objetivo de aumentar a eficiência do emprego das matérias primas, energia e água, reduzindo os riscos ambientais e aumentando a economia (Cabeda, 1999).

A piscicultura integrando um sistema de produção ao tratamento de efluentes é uma concepção moderna de aproveitamento de água, energia e infra-estrutura. Os atuais sistemas de tratamento de esgotos minimizam os problemas de saúde publica e os impactos ambientais, mas não fazem integração com sistemas produtivos, tem fluxo linear e finalidade única, produzindo somente custos para o tratamento dos resíduos.

A criação de peixes integrada ao sistema de tratamento por lagoas de estabilização tem um grande potencial. O sistema tem boa eficiência na redução de patógenos e produz grande quantidade de plâncton, alimento para peixes.

A utilização de esgoto doméstico pela aqüicultura tem suas primeiras experiências relatadas por Hoer em 1904 e em 1911 na Alemanha (Ghosh et al., 1999). O sistema de criação de peixes utilizando águas servidas de lagoas de estabilização de Calcutá na Índia, talvez o maior do mundo no reuso com aqüicultura, supre 16\% do total do peixe comercializado localmente (Morrice et al., 1998).

$\mathrm{Na}$ estação de tratamento de esgotos as características do esgoto influente e as condições ambientais (temperatura, insolação, chuva etc.) não são constantes, provocando variações na qualidade do tratamento e conseqüente mudança na qualidade do efluente gerado. O sistema deve proporcionar boas condições ambientais para a vida dos peixes, para que os resultados desta piscicultura sejam expressivos, isto requer cuidados maiores do que o empregado na piscicultura tradicional.

Os sólidos suspensos analisados em microscópio são formados principalmente por algas da família das cianofíceas e clorofíceas, matéria orgânica particulada, grumos de bactérias, rotíferos e copépodos que são alimento dos peixes. Os peixes consumindo parte destes sólidos suspensos promovem a redução da DQO (Pereira, 2000).

A aqüicultura pode tornar o tratamento de efluentes uma fonte de reciclagem de água, propiciando a produção de peixes no ambiente disponibilizado pelo tratamento de efluentes, custeando a operação, além de melhorar a qualidade do efluente.

A implantação de atividades integradas com o tratamento de efluentes necessita desenvolver seus produtos com controle de qualidade para evitar riscos à saúde, por outro lado o estudo de mercado deve estabelecer se há viabilidade econômica, promovendo a sustentabilidade ambiental, social e econômica.

Neste trabalho foram estudados os principais parâmetros ambientais para avaliação da qualidade do efluente tratado, as condições para a sobrevivência e crescimento dos peixes, as possíveis limitações quanto à contaminação por patógenos dos efluentes tratados e dos peixes, a limitação quanto ao sabor do peixe produzido e as mutações genéticas nos peixes causadas por possíveis agentes genotóxicos dos efluentes tratados, analisando entraves à piscicultura com efluentes domésticos tratados para consumo humano, comparando com os peixes criados em água limpa e em uma piscicultura "tradicional" (pesque e pague $=$ testemunho).

\section{Materiais e Métodos}

Os trabalhos se realizaram em uma unidade piloto de tanques experimentais que recebiam a água da última 
lagoa de estabilização da ETE (Estação de tratamento de esgotos) continental da CASAN - Companhia de Águas e Saneamento de Santa Catarina - Florianópolis, no período de 28/11/2001 a 5/06/2002 num total de 189 dias.

O sistema de tratamento composto por uma lagoa anaeróbica e três lagoas facultativas, operava com um tempo de detenção médio de 15,3 dias (Kracik, 1998).

\section{Qualidade do efluente tratado}

Durante o período experimental, foram acompanhados os seguintes parâmetros de qualidade da água da entrada e da saída dos tanques experimentais: temperatura com termômetro de coluna de mercúrio e precisão de $0,1^{\circ} \mathrm{C}$, disco de Secchi, $\mathrm{pH}$ feitas por pHmetro Oakton, e $\mathrm{O}_{2}$ dissolvido com Oxímetro YSI 55 realizados 3 vezes por semana, enquanto os parâmetros: DQO, N-orgânico total, N-Kjedhal, N-nitrato, N-nitrito, $\mathrm{N}$-amônia, orto-fosfato, fósforo total, clorofila " $a$ " e sólidos suspensos tiveram periodicidade quinzenal. Os parâmetros: DBO, coliformes fecais e totais foram analisados com periodicidade mensal pelo Laboratório da CASAN, conforme metodologias descritas em APHA - AWWA - WPCF (1992). As análises de colimetria foram feitas utilizando o Método Colilert.

\section{Piscicultura}

Devido a rusticidade e a facilidade de informações sobre suas exigências e características, a tilápia (Oreochromis niloticus) foi a espécie de peixe utilizada no experimento.

A biometria dos peixes foi realizada mensalmente tomando-se o comprimento total com precisão de $0,1 \mathrm{~cm}$ e peso total com precisão de $0,1 \mathrm{~g}$ em balança digital Instrutherm BD - 141.

A água da última lagoa de estabilização da ETE continental de Florianópolis foi utilizada nos tratamentos $\mathrm{T}_{2}$ e $\mathrm{T}_{3}$. Os peixes foram mantidos em uma coluna de água de $60 \mathrm{~cm}$ com um volume d'água total de 1500 litros, foram utilizados nove tanques de fibra de vidro em formato "raceway", retangulares com as extremidades em semicírculo, com $2,40 \mathrm{~m}$ de comprimento e $1,20 \mathrm{~m}$ de largura, área de $2,57 \mathrm{~m}^{2}$ e $1,20 \mathrm{~m}$ de profundidade, volume útil de $3,8 \mathrm{~m}^{3}$.
A renovação, utilizando o efluente da última lagoa de estabilização do Sistema de Tratamento foi contínua e de $20 \%$ do volume/dia. A aeração utilizada foi do tipo "airlift", com dois tubos de $40 \mathrm{~mm}$, inclinados a $45^{\circ}$, suficiente para manter a massa d'água em movimento evitando estratificações térmicas no ambiente restrito à ação do vento. $\mathrm{O}$ fornecimento de ar foi feito por dois compressores de ar com diafragma de borracha.

O desenho experimental foi totalmente casualisado com 3 tratamentos e 3 repetições conforme Tabela 1 .

TABELA 1: Descrição dos tratamentos utilizados no experimento.

\begin{tabular}{clc}
\hline $\begin{array}{c}\text { Série de } \\
\text { tanques }\end{array}$ & \multicolumn{1}{c}{ Descrição } & $\begin{array}{c}\text { Densidade } \\
\text { dos peixes }\end{array}$ \\
\hline $\mathrm{T}_{1}$ & Água limpa + ração & 7 peixes $/ \mathrm{m}^{2}$ \\
$\mathrm{~T}_{2}$ & $\begin{array}{l}\text { Efluente da lagoa estabilização } \\
\text { s/ração }\end{array}$ & 3 peixes $/ \mathrm{m}^{2}$ \\
& $\begin{array}{l}\text { Efluente da lagoa estabilização } \\
\mathrm{T}_{3}\end{array}$ & 7 peixes $/ \mathrm{m}^{2}$ \\
\hline
\end{tabular}

A água limpa utilizada no $T_{1}$ foi proveniente da rede de distribuição da CASAN, com o objetivo de neutralizar o cloro ativo existente na rede de distribuição, a água passou previamente por um tanque intermediário com aeração e tempo de detenção de 3,6 dias. A ração utilizada no $T_{1}$ foi Aqua Supra (Guabi) para engorda de peixes, com $28 \%$ de proteína bruta.

As diferentes densidades utilizadas no experimento $\left(\mathrm{T}_{2}\right.$ e $\left.\mathrm{T}_{3}\right)$ possibilitaram testar a capacidade de produzir tilápias com tamanho comercial $(350 \mathrm{~g})$. Também permitiu analisar a influência da densidade na qualidade do efluente e se a capacidade de produção (biomassa produzida) está sendo limitada pelo número de peixes.

\section{Qualidade do pescado}

A carne do pescado produzido no efluente de lagoas de estabilização foi analisada quanto aos patógenos existentes na carne e na pele dos peixes e quanto ao sabor (análise sensorial).

A análise de presença de patógenos na carne e na pele dos peixes foi realizada pelo Laboratório de Análises microbiológicas do Departamento de Ciência e Tecnologia dos Alimentos da Universidade Federal de Santa Catarina. Foram analisados os coliformes, 
Salmonella sp. (presença/ausência) e Staphylococcus aureus (UFC - unidades formadoras de colônias) da pele e da carne dos peixes criados com água do efluente de Lagoas de estabilização, na água potável e de um "testemunho" (peixes de uma piscicultura comercial localizada no município de Paulo Lopes). Os peixes foram transportados vivos e sacrificados no laboratório por secção da coluna cervical, para a análise da pele foi utilizada a técnica de SWAB. A análise do músculo foi realizada a partir de filés dos peixes utilizando a técnica de tubos múltiplos, estas análises seguiram as metodologias descritas em APHA (2001).

A análise sensorial comparativa entre os peixes criados no efluente e na água limpa com ração foi realizada pelo Laboratório de Análise Sensorial do Departamento de Ciência e Tecnologia de Alimentos da Universidade Federal de Santa Catarina segundo o método de Análise Descritiva Quantitativa - ADQ (ABNT, 1985) utilizando descritores do "flavors" desenvolvidos conforme as recomendações do método.

A escala utilizada para obtenção dos valores foi a seguinte:

$0=$ Ausente, não perceptível.

$1=$ Muito leve, muito levemente perceptível.

$2=$ Leve, levemente perceptível.

$3=$ Perceptível

$4=$ Forte, intenso.

$5=$ Muito forte, muito intenso.

As amostras de tilápias foram analisadas por julgadores treinados do Laboratório de Análise Sensorial, após serem descongeladas em geladeira a $6 \pm 2^{\circ} \mathrm{C}$ por $24 \mathrm{~h}$, filetados e cozidos em microondas. As amostras foram servidas uma a uma com números de identificação aleatórios de três dígitos.

\section{Teste de mutações genéticas nos peixes}

O teste de genotoxicidade mede os danos cromossômicos causados às células sangüíneas dos peixes. Os contaminantes químicos (agentes genotóxicos), em doses subletais causam aberrações genéticas detectáveis com a modificação no DNA, produzindo núcleos secundários: os micronúcleos.
A contagem dos micronúcleos nos eritrócitos, células sangüíneas, foi realizada em microscópio ótico (Olympus BX4O acoplado a um microcomputador Maxtech - Pentium 100 com o programa de análise de imagem - Imagem Pró Plus) com resolução 10 x 100 . O software Imagem Pró Plus permitiu medir o tamanho dos micronúcleos e núcleos. Os micronúcleos são 1/10 a $1 / 3$ menores do que o núcleo conforme descrito por Carrano e Natarajan (2002). A análise de micronúcleos foi realizada pelo LABTOX - Laboratório de Toxicologia Ambiental do Departamento de Engenharia Sanitária e Ambiental da Universidade Federal de Santa Catarina

Foram analisados os peixes criados no efluente da lagoa de estabilização, em água potável e proveniente de uma piscicultura comercial localizada no município de Paulo Lopes, as amostras constaram de cinco indivíduos para cada ambiente. Os peixes chegaram vivos ao laboratório onde foi retirado $1 \mathrm{~mL}$ de sangue utilizando seringa heparinizada, para a contagem dos micronúcleos em um esfregaço do sangue conforme a análise da metodologia descrita.

\section{Resultados e Discussão}

\section{Biometrias}

Os resultados das análises biométricas dos peixes apresentados na Figura 1 demonstram o maior crescimento dos peixes criados em água limpa e ração $\left(\mathrm{T}_{1}\right)$ com relação aos criados em água do efluente tratado em lagoas de estabilização sem acréscimo de ração $\left(T_{2}\right.$ $\mathrm{e}_{3}$ ).

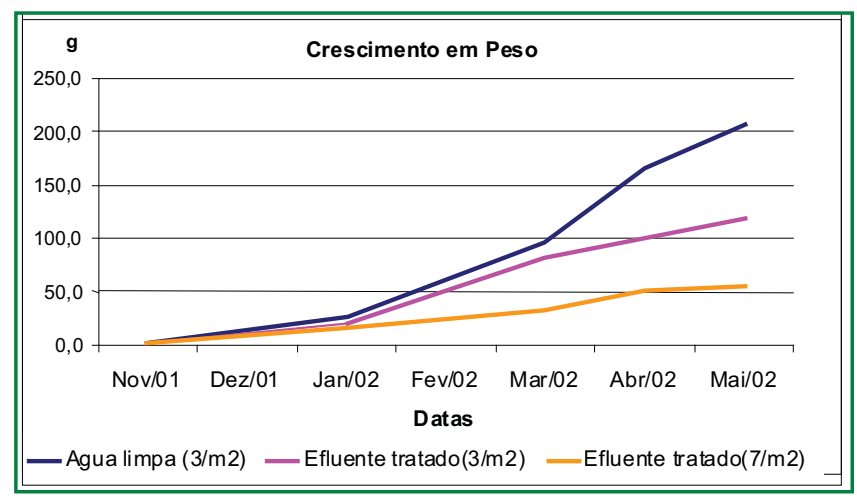

FIGURA 1: Pesos médios mensais dos peixes nos tratamentos experimentais durante o período de criação. 
$\mathrm{O}$ aumento da densidade $\left(\mathrm{T}_{2 \mathrm{x}} \mathrm{T}_{3}\right)$ produziu diferença de crescimento nos peixes. Apesar da densidade do $\mathrm{T}_{3}$ ser maior do que o dobro do $\mathrm{T}_{2}\left(3 \mathrm{e} 7 / \mathrm{m}^{2}\right)$ a produção não apresentou diferença significativa (ANOVA - Teste Tuckey $\mathrm{p}>0,05$ ) entre os tratamentos $\mathrm{T}_{3}$ e $\mathrm{T}_{2}$ conforme pode ser observado na Tabela 2 .

TABELA 2: Avaliação da produtividade da criação de peixes no experimento.

\begin{tabular}{|c|c|c|c|c|}
\hline & $\begin{array}{l}\text { Peso } \\
\text { final }\end{array}$ & $\begin{array}{l}\text { Desvio } \\
\text { Padrão }\end{array}$ & $\begin{array}{c}\text { Sobre- } \\
\text { vivência }\end{array}$ & $\begin{array}{c}\mathrm{Kg} / \mathrm{ha} / \\
\text { safra }\end{array}$ \\
\hline Água limpa (7/m²) & 204,0 & 31,3 & $88 \%$ & 12.612 \\
\hline Efluente tratado $\left(3 / \mathrm{m}^{2}\right)$ & 126,9 & 14,0 & $100 \%$ & 3.807 \\
\hline Efluente tratado $\left(7 / \mathrm{m}^{2}\right)$ & 60,9 & 3,9 & $92 \%$ & 3.936 \\
\hline
\end{tabular}

Os resultados obtidos no experimento, descritos na Tabela 2, podem ser considerados bons quando comparados aos de Leon e Moscosso (1999) consideram que a máxima capacidade de carga para produzir tilápias em lagoas de estabilização é de $4.400 \mathrm{~kg} / \mathrm{ha}$, sem o uso de alimento suplementar. Os autores consideram que acrescentar ração não aumenta a capacidade de produção de peixes devido à alta produtividade do ambiente.

Resultados como os relatados por Pillay (1993) que considera alta a produção de $1.300 \mathrm{~kg} / \mathrm{ha}$. de tilápias obtidas em viveiros adubados e de Boll et al. (2002) que obtiveram produções de $2.300 \mathrm{~kg} / \mathrm{ha}$. em sistemas de policultivo de tilápias e carpas utilizando dejetos de suínos para fertilizar a água, podem ser considerados comparáveis aos do presente estudo.

No entanto, Zimmermann e Fitzsimmons (2004) descrevem criações semi-intensivas de tilápias com produções de 2.500 a $12.500 \mathrm{~kg}$. ha ${ }^{-1}$. safra $^{-1}$ obtidos com fertilização e alimentação suplementar, sem limitações quanto à qualidade de água, condições desejáveis para o cultivo.

\section{Qualidade da água nos tanques ex- perimentais para a sobrevivência dos peixes}

Durante o período experimental as condições para sobrevivência dos peixes quanto ao $\mathrm{pH}$, oxigênio dissolvido e temperatura estiveram dentro de parâmetros suficientes para a criação de peixes, fato confirmado pela alta sobrevivência na maioria dos tanques experimentais conforme a Tabela 3.

TABELA3: Parâmetros ambientais máximos e mínimos durante o período experimental.

\begin{tabular}{c|cccccccc}
\hline & $\begin{array}{c}\text { Transparên- } \\
\text { cia }(\mathbf{c m})\end{array}$ & pH & \multicolumn{3}{c}{$\begin{array}{c}\text { Oxigênio } \\
(\mathbf{m g} / \mathbf{L})\end{array}$} & \multicolumn{2}{c}{$\begin{array}{c}\text { Temperatura } \\
{ }^{\circ} \mathbf{C}\end{array}$} \\
\hline & Max & Min & Max & Min & Max & Min & Max & Min \\
$\mathbf{T}_{1}$ & $>60$ & 10 & 9,2 & 5,3 & 13,6 & 1,5 & 30,9 & 18,1 \\
$\mathbf{T}_{2}$ & 50 & 11 & 10,1 & 6,3 & 18,7 & 1,6 & 30,7 & 18,2 \\
$\mathbf{T}_{3}$ & 50 & 11 & 10,1 & 6,3 & 18,0 & 1,7 & 31,0 & 18,3 \\
\hline
\end{tabular}

Os resultados das analises de $\mathrm{pH}$ e oxigênio dissolvido apresentaram grande amplitude. Pereira (2000) estudando este ambiente de lagoas de estabilização, observou problemas para os peixes no período da noite devido às baixas concentrações de oxigênio dissolvido, baseados neste estudo foram utilizados "airlifts" durante o experimento que mantiveram condições mínimas para a sobrevivência dos peixes, fato importante a ser considerado para estas condições e que propiciou alta sobrevivência.

A elevação do $\mathrm{pH}$ no ciclo de $24 \mathrm{~h}$ aumenta o percentual de $\mathrm{N}$ - amônia não ionizada $\left(\mathrm{NH}_{3}\right)$, tóxica para os peixes. Em função desta possibilidade as concentrações de $\mathrm{N}$ - amônia total deverão estar abaixo de $2,0 \mathrm{mg} / \mathrm{L}$ como margem de segurança para o bom crescimento e sobrevivência dos peixes (Vinatea, 2004).

No tratamento $\mathrm{T}_{1}$, que utilizou água limpa, as concentrações de $\mathrm{N}$ - amônia estiveram em mais de $90 \%$ das amostras coletadas abaixo de 2,0mg/L.

Para os tratamentos $\mathrm{T}_{2}$ e $\mathrm{T}_{3}$ em apenas $10 \%$ das amostras coletadas as concentrações foram favoráveis ao crescimento dos peixes. Os distúrbios orgânicos desta intoxicação se refletem na falta de apetite e dificuldades para respirar, estes sintomas sub-letais dificultam a vida dos peixes dificultando a alimentação e por conseguinte o crescimento (Vinatea, 2004).

A Tabela 4 mostra os valores médios e desvio padrão dos compostos nitrogenados dos tratamentos experimentais e do efluente da lagoa de estabilização que entrou nos tanques de criação de peixes, dos tratamentos 
$\mathrm{T}_{2}$ e $\mathrm{T}_{3}$. O sistema de criação de peixes criou condições que reduziram as concentrações médias de Nitrogênio orgânico e N-Kjedahl com relação ao efluente tratado da lagoa de estabilização que entrou nos tanques com peixes. A eficiência de remoção do $\mathrm{T}_{2}$ foi de $41 \%$ para o N - orgânico e de $46 \%$ para o $\mathrm{N}$ - Kjedahl, enquanto o $\mathrm{T}_{3}$ apresentou a remoção de $47 \%$ de $\mathrm{N}$ - orgânico e de $47 \%$ do N - Kjedahl.

Nos dados apresentados na Tabela 4, o N - amônia foi a forma inorgânica mais representativa do Nitrogênio no efluente da lagoa de estabilização que alimentou os tanques experimentais. Nos tratamentos $\mathrm{T}_{2}$ e $\mathrm{T}_{3}$ parte deste $\mathrm{N}$ - amônia foi nitrificado a $\mathrm{N}$ - nitrito e $\mathrm{N}$ - nitrato, evidenciado pelas maiores concentrações de $\mathrm{N}$ - nitrito e N - nitrato nos tanques experimentais e concentrações mais baixas de $\mathrm{N}$ - amônia do que no efluente da lagoa de estabilização.

TABELA 4: Valores médios (Méd) em mg/L e desvio padrão (DP) da série nitrogenada dos tratamentos experimentais e do efluente da lagoa de estabilização.

\begin{tabular}{c|lllllllllll}
\hline \multirow{2}{*}{$\begin{array}{c}\text { Trata- } \\
\text { mentos }\end{array}$} & $\begin{array}{l}\text { N-Orgâ- } \\
\text { nico total }\end{array}$ & $\begin{array}{c}\text { N-Kje- } \\
\text { dahl }\end{array}$ & \multicolumn{2}{c}{ Nitrato } & \multicolumn{2}{c}{ Nitrito } & Amônia \\
\cline { 2 - 3 } & Méd & DP & Méd & DP & Méd & DP & Méd & DP & Méd & DP \\
\hline T1 & 10,0 & 5,9 & 11,7 & 5,4 & 0,1 & 0,2 & 0,1 & 0,1 & 1,3 & 1,3 \\
T2 & 16,0 & 3,5 & 23,0 & 8,2 & 3,3 & 1,5 & 1,9 & 1,7 & 6,8 & 4,7 \\
T3 & 14,4 & 4,5 & 22,4 & 7,1 & 3,0 & 0,8 & 2,5 & 2,0 & 7,7 & 4,3 \\
Lagoa & 27,1 & 3,2 & 42,3 & 6,6 & 0,2 & 0,5 & 0,1 & 0,1 & 19,7 & 5,6 \\
\hline
\end{tabular}

A grande quantidade de nutrientes e a pouca profundidade dos tanques experimentais, proporcionaram boas condições para a proliferação de plâncton, evidenciada pela maior concentração inicial dos sólidos suspensos totais com relação ao efluente da lagoa de estabilização que abastecia os tanques.

As regressões lineares das análises de sólidos suspensos dos tratamentos $\mathrm{T}_{2}$ e $\mathrm{T}_{3}$ durante o período experimental, evidenciaram uma diminuição das concentrações máximas do início do experimento, para as concentrações mínimas na fase final do experimento. $\mathrm{O}$ crescimento dos peixes e conseqüente aumento do consumo de alimento (sólidos suspensos) podem ser considerados como as causas desta tendência (Figura 2).

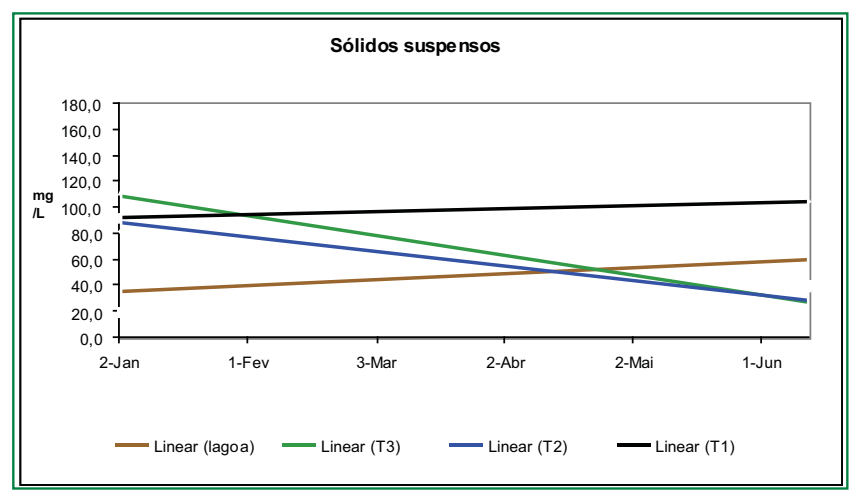

FIGURA 2: Regressão linear das concentrações de sólidos suspensos dos tratamentos experimentais T1, T2, T3 e do efluente da lagoa de estabilização durante o período de criação dos peixes.

As regressões lineares do $T_{1}$ e do efluente da lagoa de estabilização das concentrações de sólidos suspensos apresentaram variações menores entre as mínimas e máximas, com tendência de aumento no final do experimento. No $\mathrm{T}_{1}$ o aumento provavelmente foi devido à elevação do consumo de ração pelos peixes e conseqüente aumento da excreção de fezes e restos de ração.

Na Tabela 5, estão apresentados os valores médios referentes ao fósforo (ortofosfatos e fósforo total), a carga orgânica (DBO, DQO e sólidos suspensos) caracterizando um ambiente muito eutrofizado. (CONAMA, 2005)

Os valores médios máximos e mínimos da contaminação por coliformes fecais apresentados na Tabela 5, mostraram que os tanques experimentais estiveram durante todo o período experimental quantidades menores do que $10^{3} \mathrm{NMP} / 100 \mathrm{~mL}$ de coliformes fecais, padrões exigidos para a criação de peixes pela WHO (1989) e pela Resolução do CONAMA No. 274, de 2000 (CONAMA, 2005).

O efluente tratado apresentou apenas uma amostra acima do padrão $\left(2,4 \times 10^{3} \mathrm{NMP} / 100 \mathrm{~mL}\right)$ ficando as demais amostras com valores aceitos pela legislação.

As concentrações médias de clorofila $a$ nos tanques experimentais e no efluente da lagoa de estabilização, apresentados na Tabela 6 , indicam que a produtividade primária obtida poderia ter sido ainda maior, visto que são menores do que as médias entre $0,73-1,11 \mathrm{mg} / \mathrm{m}^{3}$ 
de Cla, obtidas por Leon e Moscosso (1999) trabalhando com criação de tilápias com efluente de lagoas de estabilização no Perú,

TABELA 5: Valores médios em $\mathrm{mg} / \mathrm{L}$ do fósforo total, ortofosfatos, DBO, DQO e sólidos suspensos, com a respectiva média (Méd) desvio padrão (DP) e os valores médios máximos e mínimos de coliformes fecais em NMP/100mL obtidos durante o período experimental nos tanques experimentais e no efluente da lagoa de estabilização.

\begin{tabular}{c|ccccccccccccc}
\hline & $\begin{array}{c}\text { Fosfato } \\
\text { total }\end{array}$ & $\begin{array}{c}\text { Ortofos- } \\
\text { fatos }\end{array}$ & DBO & \multicolumn{2}{c}{ DQ0 } & \multicolumn{3}{c}{ S. Susp. Col. fecais } \\
\cline { 2 - 11 } & Méd & DP & Méd & DP & Méd & DP & Méd & DP & Méd & DP & Max & Min \\
\hline T1 & 4,3 & 1,4 & 6,4 & 4,9 & 32,7 & 6,1 & 169,9 & 68,1 & 96,9 & 23,7 & 18 & 1 \\
T2 & 9,0 & 2,0 & 11,0 & 2,8 & 37,3 & 7,7 & 163,3 & 56,6 & 61,8 & 31,1 & 93 & 2 \\
T3 & 9,7 & 2,8 & 11,6 & 2,6 & 42,4 & 6,3 & 186,3 & 78,3 & 73,1 & 32,1 & 144 & 4,2 \\
Lag0a & 10,0 & 2,8 & 11,2 & 4,8 & 41,6 & 15,4 & 199,6 & 103 & 45,4 & 18,8 & 2400 & 3 \\
\hline
\end{tabular}

Segundo a classificação do CONAMA (2005) as águas dos tanques experimentais e do efluente da lagoa de estabilização estariam enquadradas na Classe 3 , águas com padrão para utilização em aqüicultura, com relação à clorofila "a".

TABELA 6: Concentrações médias de clorofila $a$ em $\mathrm{mg} / \mathrm{m}^{3}$ dos tratamentos experimentais e do efluente de lagoas de estabilização.

\begin{tabular}{c|c|c}
\hline Tanque & Médias & Desvio padrão \\
\hline $\mathrm{T} 1$ & 0,7 & 0,6 \\
\hline $\mathrm{T} 2$ & 0,5 & 0,3 \\
\hline $\mathrm{T} 3$ & 0,4 & 0,3 \\
\hline Lagoa & 0,2 & 0,2 \\
\hline
\end{tabular}

Análise comparativa da qualidade do pescado produzido no efluente de lagoas de estabilização e em água limpa

\section{Análise microbiológica}

O consumo de pescados produzidos com água de esgotos domésticos pode representar perigo à saúde, já que contém elevadas cargas de agentes microbianos patogênicos, porém considerando-se que o sistema de tratamento garante a qualidade adequada à remoção destes microrganismos, pode-se considerar não haver riscos de infecção. No entanto, o simples fato de utilização do esgoto, mesmo tratado, para a piscicultura representa um risco potencial (Bastos et al., 2003).
A OMS/WHO (Organização Mundial da Saúde) propõe a diretriz sanitária para a piscicultura de $\leq 10^{3}$ $\mathrm{CF} / 100 \mathrm{~mL}(\leq 1000$ Coliformes fecais/ $100 \mathrm{~mL}$ ) para a água dos tanques de piscicultura ou $10^{4} \mathrm{CF} / 100 \mathrm{~mL}$ na água afluente ao tanque de piscicultura, além da ausência de ovos de helmintos (tremadoides) (WHO, 1989).

O resultado das análises da pele dos peixes criados no pesque pague (testemunho), na água limpa (potável) e no efluente de lagoas de estabilização estão apresentados na Tabela 7 para comparação e análise.

Para os peixes em geral a legislação propõem o máximo de $10^{5}-10^{7}$ UFC (unidades formadoras de colônias)/g de bactérias heterotróficas e $10^{1}-10^{2}$ E. coli/g. As bactérias heterotróficas são propostas como indicadores auxiliares da qualidade da água e dos músculos, mas é discutível já que os ambientes eutrofizados podem produzir densidades elevadas de bactérias heterotróficas sem necessariamente um significado sanitário (Edwards, 1992).

TABELA 7: Resultado das análises microbiológicas da pele dos peixes criados em um pesque pague (testemunho), na água limpa, e no efluente de lagoas de estabilização.

\begin{tabular}{l|ccc}
\hline & $\begin{array}{c}\text { Peixes do } \\
\text { pesque } \\
\text { pague }\end{array}$ & $\begin{array}{c}\text { Peixes da } \\
\text { água limpa }\end{array}$ & $\begin{array}{c}\text { Peixes do } \\
\text { efluente } \\
\text { tratado }\end{array}$ \\
\hline${\text { Coliformes a } 45^{\mathbf{0}} \mathbf{C}^{\mathbf{1}}}^{-}$ & $<10$ & $<0,3-<1,5$ & $<0,3$ \\
Salmonella spp. $^{1}$ & Ausencia & Ausência & Ausência \\
Staphylococcus aureus $^{\mathbf{1}}$ & $<100$ & $<10$ & $<10$ \\
\hline
\end{tabular}

${ }^{1}$ As unidades utilizadas para a análise da qualidade da pele dos peixes foram Unidades Formadoras de Colônia/peixe (UFC/peixe) para os parâmetros Coliformes e Staphylococcus aureus; e para Salmonella sp. presença ou ausência em $25 \mathrm{~g}$ de pele dos peixes.

Na Tabela 8, estão apresentados os resultados da qualidade microbiológica dos músculos dos peixes. Segundo o certificado de análise emitido pelo Laboratório de Análise do Núcleo de Microbiológia em Alimentos da UFSC todas as amostras satisfizeram os padrões de qualidade para o consumo quanto aos parâmetros analisados, considerados como suficientes pelas autoridades sanitárias. 
TABELA 8: Resultados da análise de qualidade microbiológica dos músculos dos peixes criados no pesque pague (testemunho), na água limpa (potável) e no efluente de lagoas de estabilização.

\begin{tabular}{|c|c|c|c|}
\hline & $\begin{array}{c}\text { Peixes do } \\
\text { pesque } \\
\text { pague }\end{array}$ & $\begin{array}{c}\text { Peixes } \\
\text { da água } \\
\text { limpa }\end{array}$ & $\begin{array}{c}\text { Peixes do } \\
\text { efluente } \\
\text { tratado }\end{array}$ \\
\hline Coliformes a $45^{\circ} \mathrm{C}^{2}$ & $<3,0$ & $<3,0$ & $<3,0$ \\
\hline Salmonella spp. ${ }^{2}$ & Ausência & Ausência & Ausência \\
\hline Staphylococcus aureus $^{2}$ & $<100$ & $<100$ & $<100$ \\
\hline
\end{tabular}

${ }^{2}$ As unidades utilizadas nas análises da qualidade da carne dos peixes foram: Numero Mais Provável por grama (NMP/g) para os Coliformes, Unidades Formadoras de Colônia/grama (UFC/g) para Staphylococcus aureus; e para Salmonella sp. presença ou ausência em $25 \mathrm{~g}$ de carne dos peixes.

\section{Análise sensorial}

As amostras de tilápias (Oreochromis niloticus) foram analisadas por julgadores treinados que utilizaram descritores semelhantes aos utilizados por Van der Ploeg (1992) que considera aceitáveis os "flavors" a milho, a soja e grãos; a manteiga, a gordura; e a galinha, como características positivas, mas que não devem serem muito intensos. Os "flavors" a óleo de peixe e a camarão, lagosta são considerados de aceitação intermediária. Os "flavors" metálicos, grama/folhas, madeira, mofo/ azedo, e terra e lodo são considerados repulsivos e indesejáveis, pois são associados à decomposição do produto, provocando rejeição do produto.

A análise sensorial comparativa, encontrou diferenças de intensidade de sabor dos peixes criados no efluente tratado e dos peixes criados em água limpa, apesar das diferenças de gosto encontradas para diversos "flavors" (sabores) descritos na Figura 3 , os julgadores não foram unânimes e não tiveram opiniões que definissem significativamente se há um impedimento com relação ao sabor da carne do peixe para comercialização.

Os peixes criados com o efluente tratado de lagoas de estabilização apresentaram maior intensidade de "flavors" indesejáveis para o descritor terra/lodo, com diferença estatística significativa, conforme a Figura 3. O teste da Mínima Diferença Significativa para $\alpha=0,05$, considerou que estatisticamente as amostras são iguais com relação aos demais descritores.

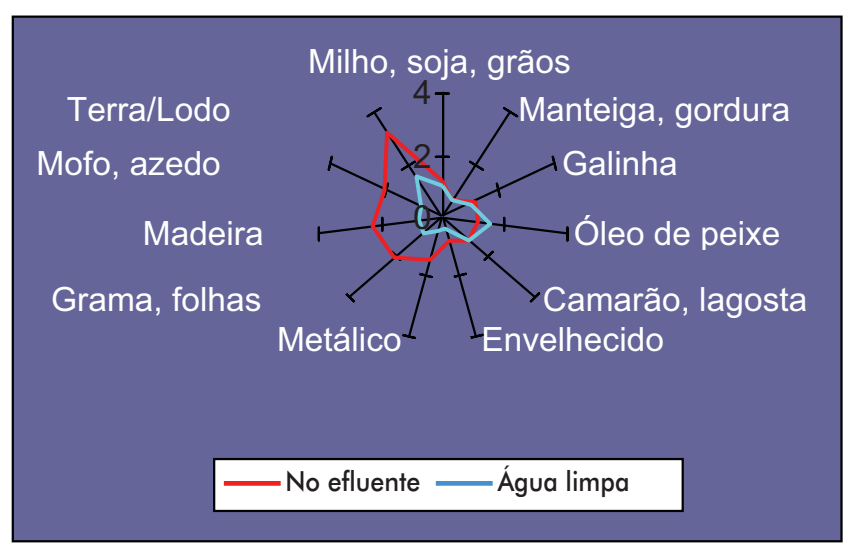

FIGURA 3: Perfil do "flavor"” definido pelos julgadores na análise sensorial da carne dos peixes criados em água limpa e no efluente de lagoas de estabilização.

3 A escala utilizada para obtenção dos valores foi a seguinte: $0=$ Ausente, não perceptível; 1 = Muito leve, muito levemente perceptível; 2 = Leve, levemente perceptível; 3 = Perceptível; 4 = Forte, intenso; 5 = Muito forte, muito intenso.

Rosa (1989) não encontrou diferenças significativas na análise de palatibilidade da carne de tilápias criadas com dejetos de suínos ou com ração de suínos, concluindo que os peixes estavam dentro dos padrões e normas para o consumo humano.

O sabor da carne dos peixes é o resultado da sua alimentação e da característica da sua carne, o sabor mais forte dos descritores do "flavors" pode ser amenizado com a manutenção dos peixes por um pequeno período em água limpa alimentando-os com ração, realizando uma depuração.

Popma e Lovshin (1996) sugerem que o período de 3 a 5 dias seriam suficientes para que os peixes mantidos em água limpa perdessem este sabor indesejado. Biato (2005) também concluiu que o tempo de 5 dias de depuração, conduzida em um tanque com água corrente e limpa, seria o ideal para permitir bons resultados para a eliminação de off flavor de tilápias criadas em viveiro com presença de geosmina, substância produtora de sabor de terra e mofo (produzida por algas cianofíceas Anabaena sp. em ambiente eutrofizado), semelhante ao encontrado nos peixes criados no efluente de lagoas de estabilização. 


\section{Teste de genotoxicidade}

A exposição dos peixes a determinadas condições ambientais extremas pode resultar na formação de células sangüíneas diferenciadas, indicando a ocorrência de uma mutação genética com a formação de eritrócito micro nucleado. O laudo da análise realizada pelo Laboratório de Ecotoxicologia da Universidade Federal de Santa Catarina do sangue dos peixes de todos os tratamentos, peixes criados em água limpa $T_{1}$ e no efluente da lagoa de estabilização $\mathrm{T}_{2} \mathrm{e} \mathrm{T}_{3}$, bem como, do testemunho utilizado (peixes de um pesque pague), relatou que não houve formação de eritrócitos micro-nucleados, indicando não haver agentes genotóxicos no efluente das lagoas de estabilização.

Os resultados apresentados neste estudo demonstram que as produções estimadas para os peixes criados no efluente de lagoas de estabilização possuem um potencial de crescimento maior do que o apresentado.

Os peixes não atingiram o peso ideal para comercialização, no entanto, a diminuição da densidade e a melhoria das condições de criação podem favorecer o crescimento dos peixes, obtendo maior tamanho e melhor qualidade para o produto (peixe) a ser comercializado.

A qualidade da carne dos peixes criados no efluente das lagoas de estabilização da ETE de Potecas é satisfatória para o consumo, conforme as análises microbiológicas e sensoriais do produto final, bem como, pela análise da qualidade do efluente utilizado durante o período experimental.

O simples fato de utilizarmos a água do esgoto, mesmo tratado, para a piscicultura representa um risco potencial para o qual devemos adotar o princípio da precaução. O sucesso deste sistema de criação de peixes depende de um trabalho criterioso de análise da qualidade do sistema de tratamento de efluentes e da qualidade do produto, peixes, de forma sistêmica (Bastos et al., 2003).

Segundo Pereira (2000) o aparecimento de algas cianofíceas é comum neste ambiente, são possíveis produtoras de toxinas, em vista disto é aconselhável o acompanhamento da presença destas toxinas nos músculos e víceras dos peixes.
O sistema de criação de peixes melhorou a qualidade do efluente da ETE, integrou atividades, reusou água e produziu peixes com algo considerado descartável, o esgoto tratado.

A melhoria da qualidade do efluente das lagoas de estabilização é desejável sob aspecto da criação dos peixes e sob o aspecto ambiental, bons motivos para novos investimentos no tratamento de efluentes domésticos e/ou industriais.

\section{Referências}

ABNT - Associação Brasileira de Normas Técnica. NBR 14140. 1985. Alimentos e bebidas - Análise sensorial - Teste de análise descritiva quantitativa (ADQ). ABNT, Rio de Janeiro, Brasil, $5 \mathrm{pp}$.

APHA - American Public Health Association. 2001. Compendium of methods of the microbiological examination of foods. $4^{\text {th }} \mathrm{ed}$. American Public Health Association, Washington, USA, 690pp.

APHA - AWWA - WPCF. 1992. Métodos normalizados para el análisis de aguas residuales. Ed. Diaz de Santos, S.A., Madrid, España, 1816pp.

Bastos, R. K. X.; Pereira, C. M.; Pivelli, R. P. Lapolli, F. R.; Lanna, E. A. T. 2003. Utilização de esgotos sanitários em piscicultura. In: Bastos, R. K. X. (Coords). Utilização de esgotos tratados em fertirrigação, hidroponia e piscicultura. ABES, RiMa, Rio de Janeiro, Brasil, p.193-223.

Biato, D. O. 2005. Detecção e controle do off flavor em tilápia do Nilo (Oreochromis niloticus), por meio de depuração e defumação. Dissertação de Mestrado, Escola Superior de Agricultura Luiz de Queiroz, Brasil, 105pp.

Boll, M. G.; Pereira C. M.; Ramage, D. 2002. Integrated fish culture systems: Are they efficient for waste recycling? ENVIRO 2002/ IWA World Water Congress, Melbourne Australia, 308pp.

Cabeda, M. 1999. Gerenciamento de subprodutos industriais Construção da matriz de resíduo. Dissertação de Mestrado, Universidade Federal de Santa Catarina, Brasil, 146pp.

Carrano, A. V.; Nataranjan, S. 2002. Considerations for populations monitoring using cytogenetic techniques. Mutation Research, 204: 379-406.

CONAMA, 2005. Resolução CONAMA 357/2005. Dispõe sobre a classificação dos corpos de água e diretrizes ambientais para o seu enquadramento, bem como estabelece as condições e padrões de lançamento de efluentes, e dá outras providências. Data da legislação: 17/03/2005 - Publicação DOU no 053, de 18/03/2005, p.5863. Disponível em $<$ http://www.mma.gov.br/port/conama/legiabre. cfm?codlegi=459> . Acesso em 02 de março de 2008.

Edwards, P. 1992. Reuse of human wastes in aquaculture - Technical review. World Bank Water and Sanitation Program the World Bank, UNDP, Report n 2, Washington, USA, p.209-269.

Ghosh, C.; Frijns, J.; Lettinga, G. 1999. Performance of silver carp (Hypophthalmicthys molitrix) dominated integrated post treatment 
system for purification of municipal waste water in temperate climate. Elsevier Bioresource Technology, 69: 255-262.

Kracik, M. E. S. 1998. Caracterização do esgoto bruto de 5 localidades da grande Florianópolis e sistema de tratamento dos esgotos do continente: Uma contribuição para a melhoria de desempenho das lagoas de estabilização de Potecas. Dissertação de Mestrado, Universidade Federal de Santa Catarina, 130pp.

Leon, S. G.; Moscosso C. J. 1999. Tratamento e uso de águas residuárias. UFPB, Campina Grande, Brasil, 110pp.

Morrice, C.; Chowdhury, N. I.; Little, D. C. 1998. Fish markets in Calcutta. Aquaculture Asia, 3 (2): 12-14.

Pereira, C. M. 2000. Avaliação do uso de peixes planctófagos como auxiliares do tratamento de efluentes. Dissertação de Mestrado, Universidade Federal de Santa Catarina, Brasil, 85pp.

Pillay, T. V. R. 1993. Aquaculture: Principles and practices. Cambridge University, Cambridge, USA, 575pp.

Popma, T.; Lovshin, L. L. 1996. Worldwide prospects for commercial production of tilapia. International Center for Aquaculture, Alabama, USA, 23pp.
Rosa, P. V. 1989. Estudos sobre consorciação tilápias do nilo suínos. Dissertação de Mestrado, Universidade Federal de Viçosa, Brasil, 65pp.

Van der Ploeg, M.1992. Testing flavor quality of preharvest channel catfish. USA: Southern Regional Aquaculture Center, P.O. Box 197 Stoneville, Mississippi 38776, Disponível em $<$ http://govdocs. aquake.org/cgi/reprint/2003/724/7240100.pdf>. Acesso em 03 de março de 2008.

Vinatea, A. L. 2004. Princípios químicos de qualidade da água em aqüicultura: Uma revisão para peixes e camarões. Ed. UFSC, Florianópolis, Brasil, 231pp.

WHO - World Health Organization. 1989. Health guidelines for the use of wastewater in agriculture and aquaculture. Technical Report Series, $\mathbf{N}^{\mathbf{0}} \mathbf{7 7 8}$, Report of a Scientific Group, Genebra, Suíça, 75pp.

Zimmermann, S.; Fitzsimmons, K. 2004. Tilapicultura intensiva. In: Cyrino, J. E. P. (Ed.). Tópicos especiais em piscicultura de água doce tropical intensiva. TecArt, São Paulo, Brasil, p.239266. 